ANNALES

POLONICI MATHEMATICI

$94.3(2008)$

\title{
Some monotonicity and limit results for the regularised incomplete gamma function
}

\author{
by Wojciech Chojnacki (Adelaide and Warszawa)
}

\begin{abstract}
Letting $P(u, x)$ denote the regularised incomplete gamma function, it is shown that for each $\alpha \geq 0, P(x, x+\alpha)$ decreases as $x$ increases on the positive real semiaxis, and $P(x, x+\alpha)$ converges to $1 / 2$ as $x$ tends to infinity. The statistical significance of these results is explored.
\end{abstract}

1. Introduction. Euler's gamma function

$$
\Gamma(u) \triangleq \int_{0}^{\infty} t^{u-1} e^{-t} d t \quad(u>0)
$$

plays an important role in many areas of mathematics and has been widely studied. The incomplete gamma function and its complement

$$
\begin{aligned}
& \gamma(u, x) \triangleq \int_{0}^{x} t^{u-1} e^{-t} d t \quad(u>0, x \geq 0), \\
& \Gamma(u, x) \triangleq \int_{x}^{\infty} t^{u-1} e^{-t} d t
\end{aligned}
$$

and the regularised incomplete gamma function and its complement

$$
\begin{aligned}
& P(u, x) \triangleq \frac{\gamma(u, x)}{\Gamma(u)} \quad(u>0, x \geq 0) \\
& Q(u, x) \triangleq 1-P(u, x)
\end{aligned}
$$

also appear in many different contexts and applications. An extended and highly readable overview on the incomplete gamma function and the related functions can be found in [2]. For a sample of more recent work, see [3].

The aim of this paper is to prove that for each $\alpha \geq 0$, (i) $P(x, x+\alpha)$ decreases as $x$ increases on the positive real semi-axis; and (ii) $P(x, x+\alpha)$ tends to $1 / 2$ as $x \rightarrow \infty$.

2000 Mathematics Subject Classification: Primary 33B15; Secondary 62H12.

Key words and phrases: gamma function, regularised incomplete gamma function, chi-square distribution, monotonicity. 
The original motivation for these results comes from estimation theory. Suppose that the outcome of a chance experiment is described by a realvalued random variable $X$ with mean $m$ and variance $\sigma^{2}$. In the event that $m$ and $\sigma^{2}$ are unknown, these values can be estimated based on several repetitions of the experiment. If the outcomes of $n$ repetitions are represented by a sequence $X_{1}, \ldots, X_{n}$ of $n$ independent copies of $X$, then a natural estimate of $m$ is the sample mean

$$
\bar{X}_{n} \triangleq \frac{1}{n} \sum_{k=1}^{n} X_{k}
$$

and a natural estimate of $\sigma^{2}$ is the sample variance

$$
S_{n}^{2} \triangleq \frac{1}{n} \sum_{k=1}^{n}\left(X_{k}-\bar{X}_{n}\right)^{2}
$$

Sometimes the sample variance is defined as

$$
S_{n}^{\prime 2} \triangleq \frac{1}{n-1} \sum_{k=1}^{n}\left(X_{k}-\bar{X}_{n}\right)^{2}
$$

The advantage of adopting the latter expression is that it specifies a meanunbiased estimator of $\sigma^{2}$-the expected value of $S_{n}^{\prime 2}$ is equal to $\sigma^{2}$.

Assume henceforth that $X$ is normally distributed. The random variable

$$
Y_{n} \triangleq n S_{n}^{2} / \sigma^{2}=(n-1) S_{n}^{\prime 2} / \sigma^{2}=\frac{1}{\sigma^{2}} \sum_{k=1}^{n}\left(X_{k}-\bar{X}_{n}\right)^{2}
$$

then has a chi-square distribution with $n-1$ degrees of freedom [14, Chapter $8, \S 45$, Theorem 1] and its cumulative distribution function is given by

$$
\begin{aligned}
\mathrm{P}\left(Y_{n} \leq x\right) & =\frac{1}{2^{(n-1) / 2} \Gamma\left(\frac{1}{2}(n-1)\right)} \int_{0}^{x} t^{(n-1) / 2-1} e^{-t / 2} d t \\
& =P\left(\frac{1}{2}(n-1), \frac{1}{2} x\right) \quad(x \geq 0),
\end{aligned}
$$

with $\mathrm{P}(A)$ denoting the probability of the event $A$. Furthermore, in accordance with a result of van der Vaart [11], $S_{n}^{\prime 2}$ is a negatively median-biased estimator of $\sigma^{2}$ in the sense that

$$
\mathrm{P}\left(S_{n}^{\prime 2} \leq \sigma^{2}\right)>\frac{1}{2}
$$

for each $n$. Starting from the identities

$$
\mathrm{P}\left(S_{n}^{\prime 2} \leq \sigma^{2}\right)=\mathrm{P}\left((n-1) S_{n}^{\prime 2} / \sigma^{2} \leq n-1\right)=P\left(\frac{1}{2}(n-1), \frac{1}{2}(n-1)\right),
$$

van der Vaart derived inequality (1) from a more general inequality that he had established, namely,

$$
P(x, x)>\frac{1}{2}
$$

for each $x>0$. 
In light of the above, one may wonder whether $S_{n}^{2}$ is also negatively median-biased. Noting, in analogy to (2), that

$$
\mathrm{P}\left(S_{n}^{2} \leq \sigma^{2}\right)=\mathrm{P}\left(n S_{n}^{2} / \sigma^{2} \leq n\right)=P\left(\frac{1}{2}(n-1), \frac{1}{2} n\right),
$$

one may ask, more generally, whether

$$
P\left(x, x+\frac{1}{2}\right)>\frac{1}{2}
$$

holds for each $x>0$. It turns out that the answer to both these questions is in the affirmative.

Indeed, the monotonicity and limit properties of the functions $x \mapsto$ $P(x, x+\alpha), \alpha \geq 0$, that will be established below immediately imply that

$$
P(x, x+\alpha)>\frac{1}{2}
$$

for each $\alpha \geq 0$ and each $x>0$. This inequality subsumes (3) and (5) as special cases corresponding to $\alpha=0$ and $\alpha=1 / 2$.

But perhaps a more significant consequence of the afore-mentioned properties of the functions $x \mapsto P(x, x+\alpha), \alpha \geq 0$, one that relies on relations (2) and (4), is that the sequences $\left\{\mathrm{P}\left(S_{n}^{2} \leq \sigma^{2}\right)\right\}_{n=1}^{\infty}$ and $\left\{\mathrm{P}\left(S_{n}^{\prime 2} \leq \sigma^{2}\right)\right\}_{n=1}^{\infty}$ decrease and have the common limit $1 / 2$. Thus, while always non-zero, the negative median bias in $S_{n}^{2}$ and in $S_{n}^{\prime 2}$, measured by $\mathrm{P}\left(S_{n}^{2} \leq \sigma^{2}\right)-1 / 2$ and $\mathrm{P}\left(S_{n}^{\prime 2} \leq \sigma^{2}\right)-1 / 2$, respectively, systematically decreases as $n$, the number of samples, mounts, reaching in limit the value zero.

2. Monotonicity result. We first establish the following.

TheOREM 1. For each $\alpha \geq 0$, the function $x \mapsto P(x, x+\alpha)$ is decreasing on $(0, \infty)$.

Proof. Fix $\alpha \geq 0$ arbitrarily. For each $x>0$, represent

$$
Q(x, x+\alpha)=\frac{1}{\Gamma(x)} \int_{x+\alpha}^{\infty} t^{x-1} e^{-t} d t
$$

as

$$
Q(x, x+\alpha)=f_{1}(x) f_{2}(x)
$$

where

$$
\begin{aligned}
& f_{1}(x) \triangleq \frac{x^{x-1 / 2} e^{-x}}{\Gamma(x)} \\
& f_{2}(x) \triangleq x^{1 / 2-x} e^{x} \int_{x+\alpha}^{\infty} t^{x-1} e^{-t} d t .
\end{aligned}
$$

The result of the theorem will be established once we show that both $f_{1}$ and $f_{2}$ are increasing. 
That $f_{1}$ is increasing is a well-known fact and a special case of more general results (cf. [1, Theorem 2], [7, Theorem 1]). In what follows, we give a self-contained proof of the monotonicity property of $f_{1}$. We start with Binet's formula [13, p. 249]

$$
\ln \Gamma(x)=\left(x-\frac{1}{2}\right) \ln x-x+\frac{1}{2} \ln (2 \pi)+\int_{0}^{\infty}\left(\frac{1}{2}-\frac{1}{t}+\frac{1}{e^{t}-1}\right) \frac{e^{-t x}}{t} d t,
$$

which implies that

$$
\ln f_{1}(x)=-\frac{1}{2} \ln (2 \pi)-\int_{0}^{\infty}\left(\frac{1}{2}-\frac{1}{t}+\frac{1}{e^{t}-1}\right) \frac{e^{-t x}}{t} d t .
$$

Now, as we shall see shortly, the function

$$
g(t) \triangleq \frac{1}{2}-\frac{1}{t}+\frac{1}{e^{t}-1} \quad(t>0)
$$

is positive, and, for each $t>0$, the function $x \mapsto e^{-t x}$ monotonically decreases. This immediately implies the desired monotonicity result for $f_{1}$.

That $g(t)$ is positive for each $t>0$ can be seen as follows. Using the Maclaurin series expansion of $t \mapsto e^{t}$, we find

$$
\frac{1}{e^{t}-1}-\frac{1}{t}=-\frac{e^{t}-1-t}{t\left(e^{t}-1\right)}=-\frac{\frac{1}{2} t^{2}+o\left(t^{2}\right)}{t^{2}+o\left(t^{2}\right)} \rightarrow-\frac{1}{2} \quad \text { as } t \rightarrow 0,
$$

so $\lim _{t \rightarrow 0} g(t)=0$. The proof of the assertion will be complete once we show that $g$ is increasing. Now

$$
g^{\prime}(t)=\frac{1}{t^{2}}-\frac{e^{t}}{\left(e^{t}-1\right)^{2}}=\frac{\left(e^{t}-1\right)^{2}-t^{2} e^{t}}{t^{2}\left(e^{t}-1\right)^{2}} .
$$

The numerator of the rightmost term is equal to zero when $t=0$ and its derivative

$$
2\left(e^{t}-1\right) e^{t}-2 t e^{t}-t^{2} e^{t}=2 e^{t}\left(e^{t}-1-t-\frac{t^{2}}{2}\right)
$$

is positive, implying that both the numerator and $g^{\prime}(t)$ are positive for $t>0$. Thus $g(t)$ is indeed increasing for $t>0$.

The positivity of $g$ can alternatively be deduced from the representation

$$
\frac{g(t)}{t}=\sum_{n=1}^{\infty} \frac{2}{t^{2}+4 n^{2} \pi^{2}} \quad(t>0)
$$

(cf. [9, p. 64]). We also mention that the positivity of $g$ can be viewed as part of a more general result concerning the Maclaurin series expansion of $t \mapsto t /\left(e^{t}-1\right)$ (cf. [5, Theorem 3]). 
We now pass to proving that $f_{2}$ is increasing. Setting $t=x w$, we obtain

$$
\int_{x+\alpha}^{\infty} t^{x-1} e^{-t} d t=x^{x} \int_{1+\alpha / x}^{\infty} w^{x-1} e^{-x w} d w=x^{x} e^{-x} \int_{1+\alpha / x}^{\infty} e^{-x v(w)} \frac{d w}{w},
$$

where

$$
v(w) \triangleq w-\ln w-1 .
$$

It is readily verified that the function $w \mapsto v(w)$ is increasing on $[1, \infty)$ with image $[0, \infty)$. Let $t \mapsto w(t)$ be its inverse, which, of course, is an increasing function from $[0, \infty)$ onto $[1, \infty)$. For each $x>0$, let

$$
t_{x} \triangleq \frac{\alpha}{x}-\ln \left(1+\frac{\alpha}{x}\right) \text {. }
$$

Clearly, $t_{x}$ is non-negative, with $t_{x}=0$ when $\alpha=0$, and, as

$$
v\left(1+\frac{\alpha}{x}\right)=t_{x}
$$

we have

$$
w\left(t_{x}\right)=1+\frac{\alpha}{x} .
$$

In an independent step, note that differentiating the relation

$$
w(t)-\ln w(t)-1=t
$$

leads to

$$
w^{\prime}(t)=\frac{w(t)}{w(t)-1}
$$

for $t>0$. Now, the change of variable $w=w(t)$ and the subsequent change $t=s / x$ in the rightmost integral of (7) with use of (8) in between yield

$$
\begin{aligned}
\int_{1+\alpha / x}^{\infty} e^{-x v(w)} \frac{d w}{w} & =\int_{t_{x}}^{\infty} e^{-x t} \frac{w^{\prime}(t)}{w(t)} d t=\int_{t_{x}}^{\infty} e^{-x t} \frac{d t}{w(t)-1} \\
& =x^{-1} \int_{x t_{x}}^{\infty} e^{-s} \frac{d s}{w\left(\frac{s}{x}\right)-1} .
\end{aligned}
$$

Hence

$$
f_{2}(x)=x^{-1 / 2} \int_{x t_{x}}^{\infty} e^{-s} \frac{d s}{w\left(\frac{s}{x}\right)-1}
$$

or, equivalently,

$$
f_{2}(x)=\int_{0}^{\infty} 1_{\left(x t_{x}, \infty\right)}(s) h\left(\frac{s}{x}\right) s^{-1 / 2} e^{-s} d s,
$$


where $1_{E}$ denotes the characteristic function of the set $E$ and

$$
h(t) \triangleq \frac{t^{1 / 2}}{w(t)-1} \quad(t>0) .
$$

We shall next show that

(i) the function $h$ is decreasing on $(0, \infty)$;

(ii) the function $x \mapsto x t_{x}$ is non-increasing on $(0, \infty)$.

This will imply that, for each $s>0$, the function $x \mapsto h(s / x)$ is increasing on $(0, \infty)$ and the function $x \mapsto 1_{\left(x t_{x}, \infty\right)}(s)$ is non-decreasing on $(0, \infty)$. The increasing monotonicity of $f_{2}$ will then follow on account of (9).

To prove (i), it suffices to show that the function

$$
h_{1}(t) \triangleq h^{-2}(t)=\frac{(w(t)-1)^{2}}{t} \quad(t>0)
$$

is increasing. To this end, define

$$
h_{2}(t) \triangleq \frac{1}{2}(w(t)-1)^{2}-t w(t) \quad(t \geq 0) .
$$

In view of $(8)$,

$$
h_{2}^{\prime}(t)=(w-1) w^{\prime}-w-t w^{\prime}=-t w^{\prime}=-\frac{t w}{w-1}<0,
$$

so $h_{2}$ is decreasing. Since $h_{2}(0)=0$, it follows that $h_{2}(t)<0$ for each $t>0$. The latter result can be reformulated as

$$
2-\frac{(w-1)^{2}}{t w}>0
$$

for each $t>0$. Now, in view of (8),

$$
\begin{aligned}
h_{1}^{\prime}(t) & =\frac{2(w-1) w^{\prime}}{t}-\frac{(w-1)^{2}}{t^{2}}=\frac{2 w}{t}-\frac{(w-1)^{2}}{t^{2}} \\
& =\frac{w}{t}\left[2-\frac{(w-1)^{2}}{t w}\right] .
\end{aligned}
$$

This together with (11) yields $h_{1}^{\prime}(t)>0$ for each $t>0$, showing that $h_{1}$ is increasing.

To establish (ii), note that the derivative of $x \mapsto x t_{x}$ at $x>0$ is equal to

$$
\frac{\alpha}{x+\alpha}-\ln \left(1+\frac{\alpha}{x}\right) \text {. }
$$

By the mean-value theorem,

$$
\ln \left(1+\frac{\alpha}{x}\right)=\ln (x+\alpha)-\ln x=\frac{\alpha}{\xi}
$$

for some $\xi$ with $x \leq \xi \leq x+\alpha$. It is now obvious that expression (12) is non-positive, yielding the desired result. 
3. Limit result. We now prove the following.

Theorem 2. For each $\alpha \geq 0, \lim _{x \rightarrow \infty} P(x, x+\alpha)=1 / 2$.

Proof. Continuing with the notation from the proof of Theorem 1, we first calculate separately $\lim _{x \rightarrow \infty} f_{1}(x)$ and $\lim _{x \rightarrow \infty} f_{2}(x)$.

Using (6) and the fact that the integrand in (6) tends decreasingly to zero as $x$ increases to infinity, we infer from Levi's monotone convergence theorem that

whence

$$
\lim _{x \rightarrow \infty} \ln f_{1}(x)=-\frac{1}{2} \ln (2 \pi),
$$

$$
\lim _{x \rightarrow \infty} f_{1}(x)=\frac{1}{\sqrt{2 \pi}}
$$

This latter result can also be deduced from the well-known asymptotic expansion for the logarithm of the gamma function (see e.g. [9, p. 62]).

To determine the other limit, first note that

$$
\lim _{x \rightarrow \infty} x t_{x}=\alpha-\lim _{x \rightarrow \infty} x \ln \left(1+\frac{\alpha}{x}\right)=0 .
$$

As the function $x \mapsto x t_{x}$ is non-increasing on $(0, \infty)$, we see that, for each $s>0,1_{\left(x t_{x}, \infty\right)}(s)$ non-decreasingly tends to 1 as $x$ increases to infinity. Next, note that by de l'Hôpital's rule and (8),

$$
\lim _{t \rightarrow 0} \frac{(w(t)-1)^{2}}{t}=\lim _{t \rightarrow 0} 2(w(t)-1) w^{\prime}(t)=\lim _{t \rightarrow 0} 2 w(t)=2 .
$$

As $h$ (defined in (10)) is decreasing on $(0, \infty)$, we deduce that, for each $s>0$, $x \mapsto h(s / x)$ increasingly tends to $2^{-1 / 2}$ as $x$ increases to infinity. Thus, for each $s>0$, the integrand in (9) non-decreasingly tends to $2^{-1 / 2} s^{-1 / 2} e^{-s}$ as $x$ increases to infinity. An application of Levi's monotone convergence theorem now reveals that

$$
\lim _{x \rightarrow \infty} f_{2}(x)=2^{-1 / 2} \int_{0}^{\infty} s^{-1 / 2} e^{-s} d s,
$$

which jointly with

yields

$$
\int_{0}^{\infty} s^{-1 / 2} e^{-s} d s=2 \int_{0}^{\infty} e^{-u^{2}} d u=\sqrt{\pi}
$$

$$
\lim _{x \rightarrow \infty} f_{2}(x)=\sqrt{\frac{\pi}{2}}
$$

Finally, the last equality together with (13) leads to

$$
\lim _{x \rightarrow \infty} P(x, x+\alpha)=1-\lim _{x \rightarrow \infty} Q(x, x+\alpha)=1-\lim _{x \rightarrow \infty} f_{1}(x) f_{2}(x)=\frac{1}{2},
$$

establishing the theorem. 
4. Related work. We conclude with a few comments about related results reported in the literature.

Van der Vaart [11] established that for each $x>0$ the sequence $\{P(x+n$, $x+n)\}_{n=1}^{\infty}$ decreases and has limit $1 / 2$. Inequality (3) is one consequence of this result. Another, based on (2), is that the sequence $\left\{\mathrm{P}\left(S_{2 n+m}^{\prime 2} \leq \sigma^{2}\right)\right\}_{n=1}^{\infty}$ decreases when $m=0$ and $m=1$; the objects involved here are the same as in the Introduction. Note that van der Vaart's result is insufficient to infer that the sequence $\left\{\mathrm{P}\left(S_{n}^{\prime 2} \leq \sigma^{2}\right)\right\}_{n=1}^{\infty}$ decreases. However, as was already alluded to earlier, this latter result follows immediately from our Theorem 1.

Vietoris [12] proved that the sequence $\{P(n, n)\}_{n=1}^{\infty}$ decreases and the sequence $\{P(n, n-1)\}_{n=1}^{\infty}$ increases, with $1 / 2$ being the common limit of both sequences.

Van de Lune [10] and, independently, Temme [8] proved that the function $x \mapsto P(x, x-1)$ increases to $1 / 2$ on $[1, \infty)$.

Merkle [6] asserted that the function $x \mapsto P(x, x)$ is decreasing on $(0, \infty)$, but his argument to validate the statement is incorrect. Merkle represents $P(x, x)$ as $P(x, x)=p_{1}(x) p_{2}(x)$, where $p_{1}(x) \triangleq x^{x-1} e^{-x} / \Gamma(x)$ and $p_{2}(x) \triangleq$ $\gamma(x, x) x^{1-x} e^{x}$, and claims that both $p_{1}$ and $p_{2}$ are decreasing. But while the first function is decreasing [4], the second is not. Figure 1 illustrates the

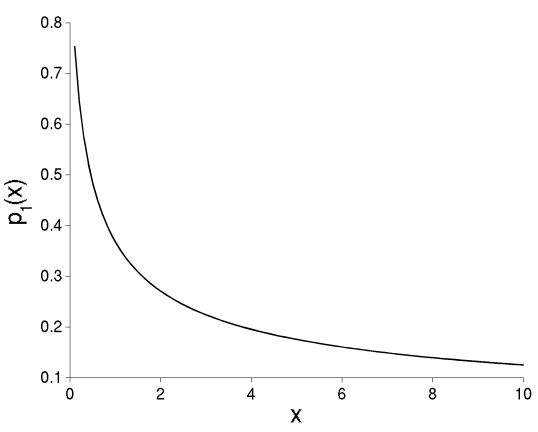

(a)

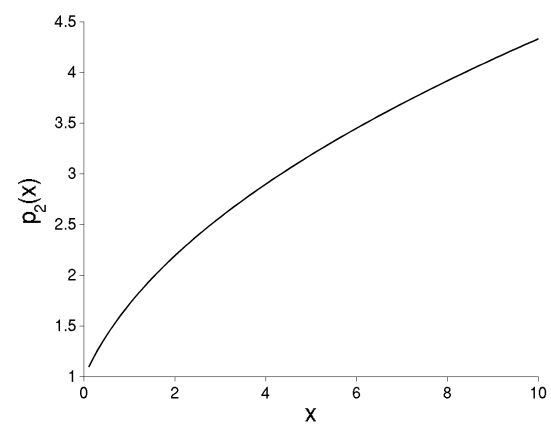

(b)

Fig. 1. Contrasting behaviours of $p_{1}$ and $p_{2}$ : (a) graph of $p_{1}$; (b) graph of $p_{2}$.

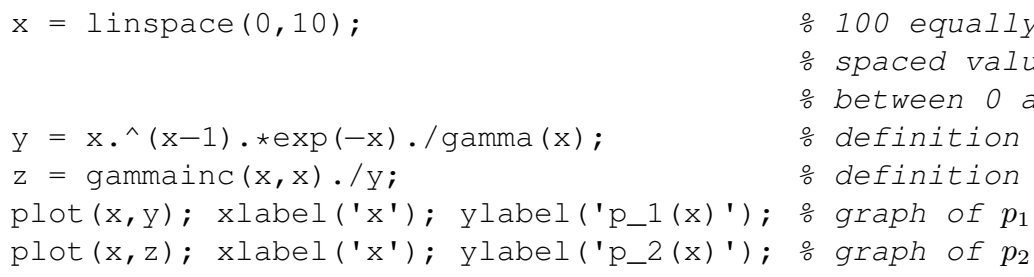

Fig. 2. Basic MATLAB code to generate graphs of $p_{1}$ and $p_{2}$. 
different behaviours of the two functions. A basic MATLAB code to generate the relevant graphs is given in Figure 2.

\section{References}

[1] H. Alzer, On some inequalities for the gamma and psi functions, Math. Comp. 66 (1997), 373-389.

[2] W. Gautschi, The incomplete gamma functions since Tricomi, in: Tricomi's Ideas and Contemporary Applied Mathematics (Rome/Turin, 1997), Atti Convegni Lincei 147, Accad. Naz. Lincei, Rome, 1998, 203-237.

[3] M. E. H. Ismail and A. Laforgia, Functional inequalities for incomplete gamma and related functions, Math. Inequal. Appl. 9 (2006), 299-302.

[4] J. D. Kečkić and P. M. Vasić, Some inequalities for the gamma function, Publ. Inst. Math. (Beograd) (N.S.) 11 (25) (1971), 107-114.

[5] S. Koumandos, Remarks on some completely monotonic functions, J. Math. Anal. Appl. 324 (2006), 1458-1461.

[6] M. J. Merkle, Some inequalities for the Chi square distribution function, Univ. Beograd. Publ. Elektrotehn. Fak. Ser. Mat. 2 (1991), 89-94.

[7] F. Qi, R.-Q. Cui, C.-P. Chen, and B.-N. Guo, Some completely monotonic functions involving polygamma functions and an application, J. Math. Anal. Appl. 310 (2005), 303-308.

[8] N. M. Temme, Some problems in connection with the incomplete gamma functions, Tech. Rep. TW 205/80, Stichting Mathematisch Centrum, Amsterdam, 1980.

[9] -, Special Functions: An Introduction to the Classical Functions of Mathematical Physics, A Wiley-Interscience Publication, Wiley, New York, 1996.

[10] J. van de Lune, A note on Euler's (incomplete) gamma function, Tech. Rep. ZN 61/75, Stichting Mathematisch Centrum, Amsterdam, 1975.

[11] H. R. van der Vaart, Some extensions of the idea of bias, Ann. Inst. Statist. Math. 32 (1961), 436-447.

[12] L. Vietoris, Dritter Beweis der die unvollständige Gammafunktion betreffenden Lochschen Ungleichungen, Österreich. Akad. Wiss. Math.-Natur. Kl. Sitzungsber. II 192 (1983), 83-91.

[13] E. T. Whittaker and G. N. Watson, A Course of Modern Analysis, 4 ed., Cambridge University Press, 1927.

[14] S. Zubrzycki, Lectures in Probability Theory and Mathematical Statistics, Elsevier, New York, 1973.

School of Computer Science

The University of Adelaide

Adelaide, SA 5005, Australia

E-mail: wojciech.chojnacki@adelaide.edu.au

Wydział Matematyczno-Przyrodniczy

Szkoła Nauk Ścisłych

Uniwersytet Kardynała Stefana Wyszyńskiego

Dewajtis 5, 01-815 Warszawa, Poland 